•研究报告・

\title{
川西高原三种雉类与其捕食者赤狐的空间关系
}

邹博研 ${ }^{1,2}$, 罗概 ${ }^{1,2}$, 朱博伟 ${ }^{1,2}$, 手江洪 ${ }^{1,2 *}$, 房超 ${ }^{3}$

1. 四川大学生命科学学院生物资源与生态环境教育部重点实验室, 成都 610065; 2. 四川大学生命科学学院四川省濒危野生动物保护生物 学重点实验室, 成都 610065; 3. 成都市雷雀生态环保科技有限公司, 成都 610065

摘要: 物种的空间分布会受到种间相互作用(如捕食关系等)和环境变量等多种因素共同影响。阐明环境变量和种间相互作用 对同域物种空间分布关系的影响, 对于理解群落聚集和生物多样性的维持机制至关重要。为了解川西高原常见雉类与捕食者 的空间分布关系及其驱动因素, 本研究利用2016-2018年在川西高原84个红外相机位点获得的682张目标物种的独立照片, 采 用条件型双物种占域模型(conditional two-species occupancy model)在相机位点尺度评估了在川西高原广泛分布的黄喉雉鶉 (Tetraophasis szechenyii)、血雉(Ithaginis cruentus)和白马鸡(Crossoptilon crossoptilon)与其捕食者赤狐(Vulpes vulpes)的空间分 布关系。结果显示: (1)在物种作用和环境变量的共同影响下, 赤狐和血雉(物种相互作用因子, species interaction factor, SIF = $1.31 \pm 0.14)$ 与赤狐和黄喉雉敦 $(\mathrm{SIF}=1.42 \pm 0.41)$ 在研究区域内的空间分布趋于重合，赤狐和血雉的空间关系随距河流距离的 增加呈现先重合后趋于分离的趋势, 而赤狐和黄喉雉敦的空间关系随距河流距离的增加呈现出由重合转为分离的趋势。赤狐 与白马鸡在空间分布上相互独立 $(\mathrm{SIF}=1)$ ，白马鸡的空间分布主要受环境因子影响，而赤狐对其没有影响。(2) 3种雉类的探 测率受物种作用的影响, 在相机位点尺度上赤狐的存在减少了3种雉类的探测率 $(p \mathrm{~B}>r \mathrm{~B}$ )。本研究为物种空间分布关系的研 究提供了新的案例, 也为理解物种共存机制和生物多样性保护提供了科学依据。

关键词: 空间分布; 捕食关系; 红外相机; 占域模型

刍博研，罗概，朱博伟，苒江洪，房超 (2021) 川西高原三种雉类与其捕食者赤狐的空间关系. 生物多样性, 29, 918-926. doi: 10.17520/biods.2020438. Zou BY, Luo G, Zhu BW, Ran JH, Fang C (2021) The spatial distribution relationship between three pheasant species and mutual predator, the red fox (Vulpes vulpes), on the Western Sichuan Plateau. Biodiversity Science, 29, 918-926. doi: 10.17520/biods.2020438.

\section{The spatial distribution relationship between three pheasant species and mutual predator, the red fox (Vulpes vulpes), on the Western Sichuan Plateau}

\author{
Boyan Zou ${ }^{1,2}$, Gai Luo ${ }^{1,2}$, Bowei Zhu ${ }^{1,2}$, Jianghong Ran ${ }^{1,2^{*}}$, Chao Fang ${ }^{3}$ \\ 1 Key Laboratory of Bio-Resource and Eco-Environment of Ministry of Education, College of Life Sciences, Sichuan University, \\ Chengdu 610065 \\ 2 Sichuan Key Laboratory of Conservation Biology on Endangered Wildlife, College of Life Sciences, Sichuan University, Chengdu \\ 610065 \\ 3 Chengdu Leique Ecology \& Environmental Protection Technology Co. Ltd., Chengdu 610065
}

\begin{abstract}
Aims: Multiple interspecific factors such as predator-prey dynamics and responses to different environmental variables collectively influence the spatial distribution of wildlife species. To be able to understand how these mechanisms influence community aggregation and biodiversity stability, it is crucial to understand role these factors play in impacting the formation of spatial distribution patterns among sympatric species.

Methods: Here, we investigated the spatial distribution correlations and driving factors of three pheasant species commonly seen or surveyed on the Western Sichuan Plateau. We combined a total of 682 independent photos obtained from 84 infrared camera traps from 2016 to 2018 with conditional two-species occupancy model. We then used the model operation to assess the spatial distribution relations on the camera site scale. We used this model operation for each of the three pheasant species (the buff-throated partridge Tetraophasis szechenyii, blood pheasant Ithaginis cruentus, and the white eared-pheasant Crossoptilon crossoptilon) and their predator the red fox which can also be
\end{abstract}

收稿日期: 2020-11-25; 接受日期: 2021-03-06

基金项目: 第二次青藏高原综合科学考察研究项目(2019QZKK0402)和全国第二次陆生野生动物资源调查项目

* 通讯作者 Author for correspondence. E-mail: rjhong-01@163.com 
found over a wide area in the Western Sichuan Plateau.

Results: We had two major results from our analyses. First, under the synergic-influence of species interactions and environmental variables, the spatial distribution of the red fox and the blood pheasant (species interaction factor, SIF = $1.31 \pm 0.14$ ) was similar to that of the red fox and the buff-throated partridge (SIF $=1.42 \pm 0.41$ ). Both these pairs tended to have a great overlap within the study area. Additionally, the spatial distribution between the red fox and the blood pheasant would overlap in some large areas and then spatial distribution overlapped decreased with the increasing distance to rivers. The spatial relationship between the red fox and the buff-throated partridge were exhibited a different trend, which has been a consistently falling overlap ratio tendency since the distance from camera site to rivers began to increase. The red fox and the white eared-pheasant shared independent distribution patterns with each other (SIF $=1)$. Environmental variables were a strong predictor for the spatial distribution pattern of white-eared pheasants. However, environmental variables hardly had any impact on the distribution strategy for the red fox. The second major result was that the detection probabilities for all three pheasant species was associated with the synergic-influence of species interactions. The presence of red fox practically reduced the detection probability for all three pheasant species on the site scale $(p \mathrm{~B}>r \mathrm{~B})$.

Conclusion: Results from this experiment provide a new up to date case study (with solid scientific basis) that focused on species distribution relationships, and help us understand the importance of species coexistence and biodiversity conservation.

Key words: spatial distribution; predator-prey relationships; camera traps; occupancy model

物种的时空分布及其驱动因素是生态学和保 护生物学的重要研究内容(Connell, 1983; Case \& Bolger, 1991)。环境变量和动物间的相互作用(如捕 食关系、竞争关系等)是决定物种分布的重要因素 (Ritchie et al, 2009)。捕食关系是自然生态系统中十 分重要和复杂的生态关系, 影响着捕食者和被捕食 者的空间分布。Creel和Winnie (2005)在美国西北部 的研究发现, 当狼(Canis lupus)群出现时, 马鹿 (Cervus elaphus)会通过减少在草地上的受食活动并 进入安全的林地来躲避狼的捕食。Rabelo等(2019) 对美洲豹(Panthera onca)栖息地选择的研究结果表 明猎物密度可以影响美洲豹的栖息地选择, 美洲豹 更倾向于使用其树栖类猎物红吼猴 (Alouatta seniculus) 和褐喉树懒(Bradypus variegatus)数量较 多的区域。当同一地区存在多种捕食者或多种猎物, 且捕食者对不同种类猎物的捕食偏好不同时，不同 种类猎物对捕食者空间分布的影响也不同。 Steinmetz等(2013)对虎(Panthera tigris)的研究表明, 其对生境位点的使用主要受印度野牛(Bos gaurus)、 水鹿(Cervus unicolor)、野猪(Sus scrofa)和印度鹿 (Muntiacus muntjak)等4种猎物丰富程度的影响, 由 于虎对野猪这一猎物的捕食偏好, 野猪的数量与虎 对位点的使用率存在显著正相关关系。也有研究认 为捕食者与被捕食者的空间分布关系是捕食关系 和环境因子共同作用的结果, Magle等(2014)对郊狼 (Canis latrans)与白尾鹿(Odocoileus virginianus)空
间分布的研究表明不同环境因子对郊狼和白尾鹿 位点使用情况的影响不同, 郊狼的占域率和白尾鹿 的占域率存在显著正相关关系。尽管在“生态恐惧” 的理论框架下, 白尾鹿会避开有郊狼的区域, 但由 于优质生存环境稀少的原因, 白尾鹿仍会选择有郊 狼的区域生存。Murphy等(2019)在马达加斯加对外 来捕食者与 27 个猎物物种的空间分布关系研究结 果显示, 当地居民放养的狗(Canis familiaris)、小灵 猫(Viverricula indica)、野猫(Felis silvestris)会影响当 地猎物马岛鼠(Nesomys spp.)、大马岛猬(Setifer setosus)、马岛林秧鸡(Mentocrex kioloides)的空间分 布, 且这种影响会随着环境的变化而变化。虽然已 经有一些研究, 但有关作为捕食动物主要食物来源 的雉类与捕食者间的空间分布关系及其驱动因素 的研究仍然较少。

赤狐(Vulpes vulpes)属食肉目犬科, 广泛分布于 欧亚大陆各种生境, 主要选择食物丰富度较高的植 被和生境类型(李路云等, 2014)。赤狐是川西高原常 见的捕食动物, 主要以啮齿动物、鸡形目鸟类和野 兔等为食(马勇等, 2014)。鸡形目鸟类生活史大多数 时间局限于地面, 飞行和扩散能力较弱, 是典型的 地栖型鸟类。黄喉雉㕫(Tetraophasis szechenyii)、血. 雉 (Ithaginis cruentus) 和白马鸡 (Crossoptilon crossoptilon)是川西高原具有代表性的鸡形目鸟类, 主要栖息于亚高寒地区的针阔混交林、针叶林、高 山灌从及高山草甸中, 具有相似的生活习性和资源 
需求。尽管3种雉类间存在一定的种间竞争, 但已有 研究表明雉类的种间竞争并不影响其共存模式 (Chen \& Luiselli, 2009; Wang et al, 2021)。本研究利 用红外相机数据对黄喉雉㕫、血雉和白马鸡与其捕 食者一一赤狐分别建立条件型双物种占域模型 (conditional two-species occupancy model)研究其在 相机位点尺度上的空间分布关系, 分析影响川西高 原3种雉类空间分布的生物和非生物因素, 探究 3 种 雉类与捕食者赤狐的空间分布关系及驱动因素, 为 物种空间分布关系的研究提供新的案例。

\section{研究方法}

\section{1 研究区域概况}

研究区域位于川西高原中部四川省甘孜藏族 自治州新龙县和白玉县境内, 地理坐标为 $98^{\circ} 36^{\prime}-$ $100^{\circ} 54^{\prime} \mathrm{E}, 30^{\circ} 22^{\prime}-31^{\circ} 40^{\prime} \mathrm{N}$, 海拔2,587-5,870 m。属 于高原季风气候, 昼夜温差大, 垂直气候变化显 著。植被群落主要有河谷早生灌从、针阔叶混交林、 亚高山针叶林、高山灌丛、高山草甸和流石滩(邓林 和杨海真, 2010; 游章强等, 2014)。

\section{2 红外相机布设}

根据研究区域内的地形和植被因素选择野生
动物丰富的区域，将调查区域划分为 $2 \mathrm{~km} \times 2 \mathrm{~km}$ 的 网格，在考虑交通、地形条件下，选取有森林分布 的网格内布设1-3台红外相机(图1)。相机布设在动 物痕迹较多的地点(如兽径、山脊等), 以最大限度提 高物种的探测概率，海拔范围在3,120-4,843 m。相 机固定在距地面30-60 cm的树干上, 全天 $24 \mathrm{~h}$ 工作, 参数设置为连续拍 3 张照片, 时间间隔为 $1 \mathrm{~s} 。$ 诼 每 台相机的GPS坐标(朱博伟等, 2019)。

\section{3 空间分布关系分析}

红外相机和占域模型的结合是在考虑环境条 件的情况下研究物种共存问题的一种有效手段 (Burton et al, 2015; D’Amen et al, 2018)。本研究使用 条件型双物种占域模型(Richmond et al, 2010)来探 究3种雉类和其捕食者赤狐的空间分布关系。占域 模型可以利用红外相机捕获到的物种出现或未出 现情况，在考虑不完全探测的情况下同时分析物种 相互作用和环境因子对物种空间分布的影响 (MacKenzie et al, 2004; Chen et al, 2019)。条件型双 物种占域模型包含 8 个基本参数(表 1 )。根据条件型 双物种占域模型的基本假设，我们将赤狐作为优势 物种(物种 $\mathrm{A}), 3$ 种雉类作为从属物种(物种 $\mathrm{B})$ 。有研 究表明赤狐的家域半径为1.5 km (Lesmeister et al,

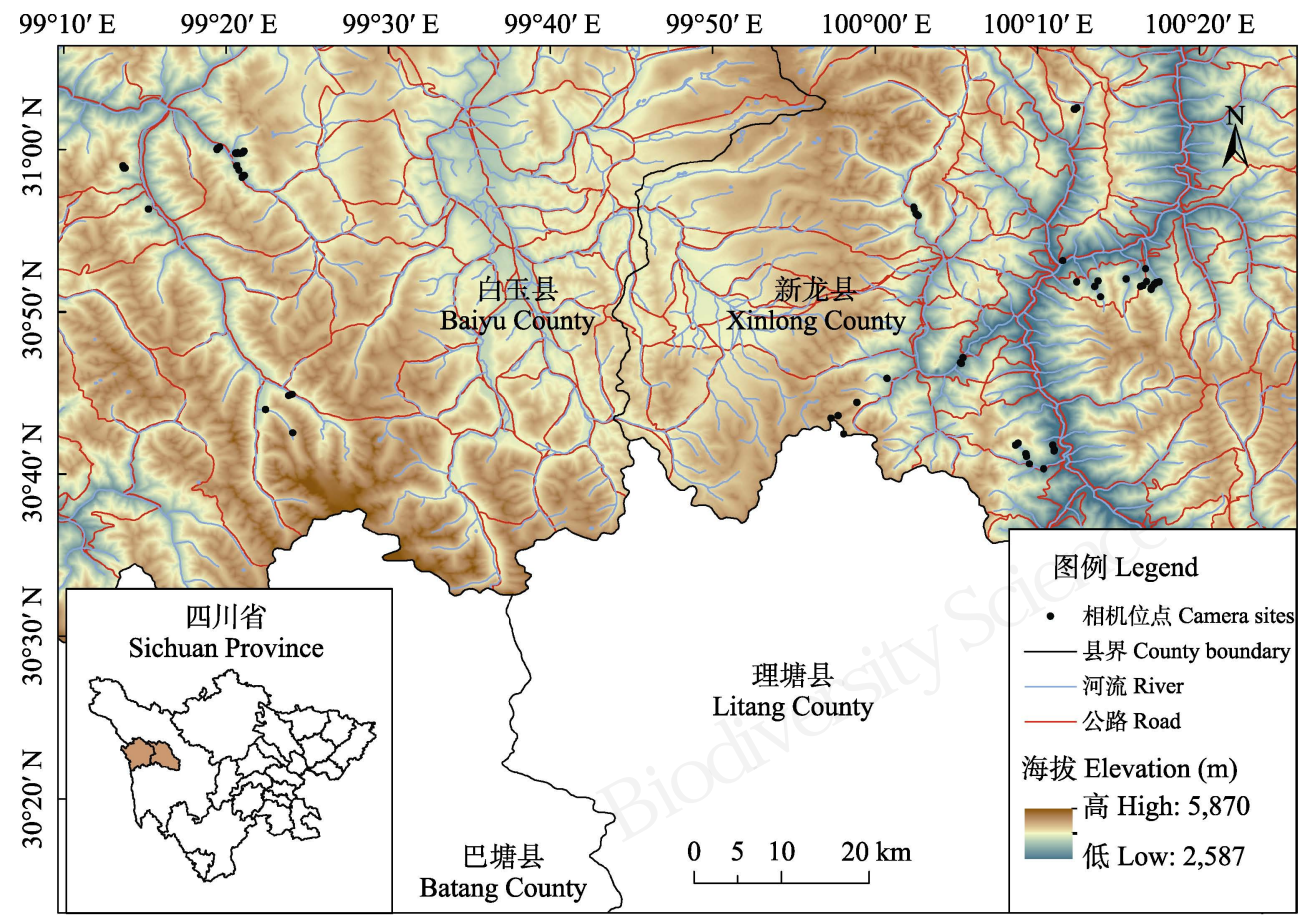

图1 研究区域位置及红外相机位点示意图

Fig. 1 The location of study area and camera sites 
表1 条件型双物种占域模型中的参数描述

Table 1 The description of parameters in conditional two-species occupancy model

\begin{tabular}{|c|c|}
\hline 参数 & 描述 \\
\hline$\underline{\text { Parameters }}$ & Description \\
\hline psiA & $\begin{array}{l}\text { 物种A对位点使用的概率 Probability of site use for } \\
\text { species A }\end{array}$ \\
\hline \multirow[t]{2}{*}{ psiBA } & 当物种A存在时, 物种B对位点使用的概率 \\
\hline & $\begin{array}{l}\text { Probability of site use for species B, given species A is } \\
\text { present }\end{array}$ \\
\hline \multirow[t]{2}{*}{ psiBa } & 当物种A不存在时, 物种B对位点使用的概率 \\
\hline & $\begin{array}{l}\text { Probability of site use for species B, given species A is } \\
\text { absent }\end{array}$ \\
\hline$p \mathrm{~A}$ & $\begin{array}{l}\text { 当物种B不存在时, 物种 } \mathrm{A} \text { 的探测概率 Probability of } \\
\text { detection for species A, given species B is absent }\end{array}$ \\
\hline$p \mathrm{~B}$ & $\begin{array}{l}\text { 当物种A不存在时, 物种B的探测概率 Probability of } \\
\text { detection for species B, given species A is absent }\end{array}$ \\
\hline$r \mathrm{~A}$ & $\begin{array}{l}\text { 当两物种都存在时, 物种A的探测概率 Probability of } \\
\text { detection for species A, given both species are present }\end{array}$ \\
\hline$r \mathrm{BA}$ & 当两物种都存在且物种 $\mathrm{A}$ 被探测到时, 物种 $\mathrm{B}$ 的探测 \\
\hline & $\begin{array}{l}\text { 概率 Probability of detection for species B, given both } \\
\text { species are present and species A is detected }\end{array}$ \\
\hline$r \mathrm{Ba}$ & 当两物种都存在且物种 $\mathrm{A}$ 未被探测到时, 物种 $\mathrm{B}$ 的探 \\
\hline & $\begin{array}{l}\text { 测概率 Probability of detection for species B, given } \\
\text { both species are present and species A is not detected }\end{array}$ \\
\hline
\end{tabular}

2015), 本研究的相机距离不大于其家域半径, 因此 我们放宽了占域模型通常需要地理封闭的假设, 定 义模型中的“占域”为“单个相机位点的使用” (Farris et al, 2016)。

以 10 天作为 1 次探测周期对研究物种分别建立 观测历史, 本次调查期间共进行了 48次探测。将海 拔、坡度、坡向、植被增强型指数 (enhanced vegetation index, EVI)、除赤狐外其他食肉动物的相 对多度指数(relative abundance index, RAI)、相机位 点距最近河流距离和相机位点距最近道路距离 7 个 变量作为占域协变量, 将每个探测周期中的具体探 测天数作为探测协变量。海拔、坡度、坡向和植被 增强型指数从地理空间数据云(http://www.gscloud. $\mathrm{cn} /$ )下载获得。河流和道路数据(国道、省道、县道、 乡道等) 从全国地理信息资源目录服务系统 (https://www.webmap.cn/)下载获得后, 使用ArcGIS 10.3测量每个相机位点到最近河流和道路的欧式距 离。除赤狐外的其他食肉动物相对多度指数为每 100 天拍摄到的物种独立照片次数(赵国静等, 2019)。为了避免出现模型不收玫问题, 我们假设在 同一个相机位点下, 优势物种的探测对从属物种的 探测不产生影响 $(r \mathrm{BA}=r \mathrm{Ba}$ ) (Wang et al, 2015), 或 者采用减少候选模型中协变量数量的方法(Murphy et al, 2019)。在构建候选模型时, 由于赤狐与黄喉雉 敦的候选模型不收玫, 因此减少了植被增强型指数
这一占域协变量而使模型收敛。

为了简化候选模型的数量, 我们首先分别对每 个物种建立单物种占域模型, 选取最优模型中的占 域和探测协变量作为每个物种的最佳占域和探测 协变量, 然后使用各物种的最佳占域和探测协变量 对 3 个物种对建立条件型双物种占域模型 (Richmond et al, 2010)。

我们分别对每个物种对建立了 8 个候选模型(附 录1), 然后用赤池信息量准则(AIC)来对模型进行 排序，同时选择 $\Delta \mathrm{AIC} \leq 2$ 且模型权重最高的模型作 为最优模型来提取各个参数并计算物种相互作用 因子 (species interaction factor, SIF) 的估计值 (Murphy et al, 2019)。物种相互作用因子表示两个物 种共同出现的关系，当SIF $=1$ 时，两物种的空间分 布相互独立; 当SIF < 1时, 两物种在相机位点相比 独立的假设下更不容易共同出现，空间分布趋于分 离; 当SIF > 1时, 两物种在相机位点相比独立的假 设下更容易共同出现，空间分布趋于重合 (Richmond et al, 2010)。所有分析均在软件 PRESENCE 2.12.43 (Hines, 2006)中完成。

\section{结果}

在2016年9月至2017年5月和2017年8月至2018 年4月期间, 共设置了 84 个相机位点, 累计监测 8,679 个相机工作日，共获得独立照片 2,817 张，其 中目标物种独立照片 682 张。拍到黄喉雉敦的相机 位点有 31 个(36.9\%), 独立照片 117 张; 拍到血雉的 相机位点有 37 个(44.1\%), 独立照片 175 张; 拍到白 马鸡的相机位点有 49 个(58.3\%), 独立照片 312 张; 拍到赤狐的相机位点有 25 个(29.8\%), 独立照片 78 张。在研究区域中还监测到其他 9 种食肉动物, 分别 为金钱豹 (Panthera pardus)、豹猫 (Prionailurus bengalensis)、猞猁(Lynx lynx)、荒漠猫(Felis bieti)、 兔狲 (Otocolobus manul) 、金 猫 (Catopuma temminckii)、狼、黄喉貂(Martes flavigula)、黄鼠 (Mustela sibirica)。

\section{1 赤狐与三种雉类的空间关系}

4 个物种的单物种占域模型结果表明，赤狐的 最优模型显示其对位点的使用概率不受占域协变 量的影响。黄喉雉敦的最优模型中占域协变量为植 被增强型指数(EVI) 和距最近河流距离; 白马鸡的 最优模型中占域协变量为海拔和距最近道路距离; 
血雉的最优模型中占域协变量为距最近河流距离 (附录2)。

条件型双物种占域模型的最优模型结果显示, 赤狐对 3 种雉类位点使用概率的影响不同，但都受 到了环境变量的影响(表2)。在环境变量的介导下, 血雉在赤狐存在时对位点的使用概率显著高于赤 狐不存在时 $(p s i \mathrm{BA}=0.81 \pm 0.30, p s i \mathrm{Ba}=0.29 \pm 0.17$, $P<0.01)$, 赤狐和血雉在研究区域内空间分布趋于 重合 $(S I F=1.31 \pm 0.14)($ 表 3$)$ 。赤狐和血雉随距河流 距离的增加, 空间关系呈现先趋于重合后趋于分离 的趋势(图2A)。黄喉雉敦在赤狐存在时对位点使用 概率也显著高于赤狐不存在时 $(p s i \mathrm{BA}=0.70 \pm 0.08$, $p s i \mathrm{Ba}=0.31 \pm 0.38, P<0.01)$ 。赤狐和黄喉雉敦在研 究区域内空间分布趋于重合 $(S I F=1.42 \pm 0.41)$ (表 3)。赤狐和黄喉雉敦的空间关系随着距河流距离的 增加呈现出由重合转为分离的趋势(图2B)。

赤狐的存在并不影响白马鸡对于位点的使用 (psiBA $=p s i \mathrm{Ba}=0.68 \pm 0.20)$, 白马鸡与赤狐在研究 区域内空间分布相互独立 $(S I F=1)$ 。白马鸡对位点 的使用概率主要受环境因子影响，随海拔升高而降 低, 随距最近道路距离升高而升高(图3)。

\section{2 赤狐对 3 种雉类探测率的影响}

单物种占域模型最优模型显示 4 个物种的探测 率均不受探测协变量的影响(附录2), 所以在建立条

表2 赤狐与 3 种雉类的条件型双物种占域模型选择结果

Table 2 Selection results of conditional two-species occupancy models for red fox and three pheasant species

\begin{tabular}{|c|c|c|c|c|}
\hline 模型 Models & 参数数量 & Number of parameters AIC & $\triangle \mathrm{AIC}$ & AIC Wt \\
\hline \multicolumn{5}{|l|}{ 赤狐 + 白马鸡 Vulpes vulpes + Crossoptilon crossoptilon } \\
\hline$p s i \mathrm{~A}, p s i \mathrm{~B}(\mathrm{ELE}+\mathrm{DTR}) ; p \mathrm{~A}, p \mathrm{~B}, r \mathrm{~B}$ & 7 & $1,120.73$ & 0 & 0.9731 \\
\hline$p s i \mathrm{~A}, p s i \mathrm{~B} ; p \mathrm{~A}, p \mathrm{~B}, r \mathrm{~B}$ & 5 & $1,128.81$ & 8.08 & 0.0171 \\
\hline$p s i \mathrm{~A}, p s i \mathrm{BA}, p s i \mathrm{Ba} ; p \mathrm{~A}, p \mathrm{~B}, r \mathrm{~B}$ & 6 & $1,130.8$ & 10.07 & 0.0063 \\
\hline$p s i \mathrm{~A}, p s i \mathrm{~B}(\mathrm{ELE}+\mathrm{DTR}) ; p \mathrm{~A}, p \mathrm{~B}$ & 6 & $1,132.21$ & 11.48 & 0.0031 \\
\hline$p s i \mathrm{~A}, p s i \mathrm{BA}(\mathrm{ELE}+\mathrm{DTR}), p s i \mathrm{Ba}(\mathrm{ELE}+\mathrm{DTR}) ; p \mathrm{~A}, p \mathrm{~B}$ & 9 & $1,137.12$ & 16.39 & 0.0003 \\
\hline$p s i \mathrm{~A}, p s i \mathrm{~B} ; p \mathrm{~A}, p \mathrm{~B}$ & 4 & $1,141.3$ & 20.57 & 0 \\
\hline$p s i \mathrm{~A}, p s i \mathrm{BA}, p s i \mathrm{Ba} ; p \mathrm{~A}, p \mathrm{~B}$ & 5 & $1,142.01$ & 21.28 & 0 \\
\hline$p s i \mathrm{~A}, p s i \mathrm{BA}(\mathrm{ELE}+\mathrm{DTR}), p s i \mathrm{Ba}(\mathrm{ELE}+\mathrm{DTR}) ; p \mathrm{~A}, p \mathrm{~B}, r \mathrm{~B}$ & 10 & $1,151.45$ & 30.72 & 0 \\
\hline \multicolumn{4}{|l|}{ 赤狐 + 黄喉雉敦 Vulpes vulpes + Tetraophasis szechenyii } & 0.9847 \\
\hline$p s i \mathrm{~A}, p s i \mathrm{BA}, p s i \mathrm{Ba} ; p \mathrm{~A}, p \mathrm{~B}, r \mathrm{~B}$ & 6 & 823.13 & 8.78 & 0.0122 \\
\hline$p s i \mathrm{~A}, p s i \mathrm{~B}(\mathrm{DTW}) ; p \mathrm{~A}, p \mathrm{~B}, r \mathrm{~B}$ & 6 & 826.47 & 12.12 & 0.0023 \\
\hline$p s i \mathrm{~A}, p s i \mathrm{~B} ; p \mathrm{~A}, p \mathrm{~B}, r \mathrm{~B}$ & 5 & 829.01 & 14.66 & 0.0006 \\
\hline$p s i \mathrm{~A}, p s i \mathrm{BA}$ (DTW), psiBa (DTW); $p \mathrm{~A}, p \mathrm{~B}$ & 7 & 833.91 & 19.56 & 0.0001 \\
\hline$p s i \mathrm{~A}, p s i \mathrm{~B}(\mathrm{DTW}) ; p \mathrm{~A}, p \mathrm{~B}$ & 5 & 833.97 & 19.62 & 0.000 \\
\hline$p s i \mathrm{~A}, p s i \mathrm{~B} ; p \mathrm{~A}, p \mathrm{~B}$ & 4 & 836.53 & 22.18 & 0 \\
\hline$p s i \mathrm{~A}, p s i \mathrm{BA}, p s i \mathrm{Ba} ; p \mathrm{~A}, p \mathrm{~B}$ & 5 & 837.41 & 23.06 & 0 \\
\hline \multicolumn{5}{|l|}{ 赤狐 + 血雉 Vulpes vulpes + Ithaginis cruentus } \\
\hline$p s i \mathrm{~A}, p s i \mathrm{~B}(\mathrm{DTW}) ; p \mathrm{~A}, p \mathrm{~B}, r \mathrm{~B}$ & 6 & 937.74 & 2.73 & 0.1974 \\
\hline$p s i \mathrm{~A}, p s i \mathrm{BA}, p s i \mathrm{Ba} ; p \mathrm{~A}, p \mathrm{~B}, r \mathrm{Ba}$ & 6 & 942.00 & 6.99 & 0.0235 \\
\hline$p s i \mathrm{~A}, p s i \mathrm{~B} ; p \mathrm{~A}, p \mathrm{~B}, r \mathrm{Ba}$ & 5 & 944.68 & 9.67 & 0.0061 \\
\hline$p s i \mathrm{~A}, p s i \mathrm{~B}(\mathrm{DTW}) ; p \mathrm{~A}, p \mathrm{~B}$ & 5 & 968.95 & 33.94 & 0 \\
\hline$p s i \mathrm{~A}, p s i \mathrm{BA}$ (DTW), psiBa (DTW); $p \mathrm{~A}, p \mathrm{~B}$ & 7 & 972.72 & 97.71 & 0 \\
\hline$p s i \mathrm{~A}, p s i \mathrm{~B} ; p \mathrm{~A}, p \mathrm{~B}$ & 4 & 975.74 & 40.73 & 0 \\
\hline$p s i \mathrm{~A}, p s i \mathrm{BA}, p s i \mathrm{Ba} ; p \mathrm{~A}, p \mathrm{~B}$ & 5 & 977.74 & 42.73 & 0 \\
\hline
\end{tabular}

参数含义见表 $1 ; \triangle \mathrm{AIC}$ : 该模型与最小AIC模型的AIC之差; AIC Wt: 模型权重; ELE: 海拔; DTR: 距最近道路距离; DTW: 距最近河流距离。 The meaning of parameters is shown in Table 1; $\triangle$ AIC, The relative difference in AIC values compared with the top ranked model; AIC Wt, Model weight; ELE, Elevation; DTR, Distance to the nearest road; DTW, Distance to the nearest river. 
表3 赤狐与 3 种雉类条件型双物种占域模型最优模型的参数估计值(平均值 \pm 标准差)

Table 3 Parameters estimates obtained from the optimal conditional two-species occupancy model applied to red fox and three pheasants (mean $\pm \mathrm{SE}$ )

\begin{tabular}{|c|c|c|c|c|c|c|c|}
\hline 物种对 Species pairs & $p s i \mathrm{~A} \pm \mathrm{SE}$ & $p s i \mathrm{BA} \pm \mathrm{SE}$ & $p s i \mathrm{Ba} \pm \mathrm{SE}$ & $p \mathrm{~A} \pm \mathrm{SE}$ & $p \mathrm{~B} \pm \mathrm{SE}$ & $r \mathrm{~B} \pm \mathrm{SE}$ & $\mathrm{SIF} \pm \mathrm{SE}$ \\
\hline $\begin{array}{l}\text { 赤狐 }+ \text { 白马鸡 Vulpes vulpes }+ \\
\text { Crossoptilon crossoptilon }\end{array}$ & $+0.50 \pm 0.10$ & $0.68 \pm 0.20$ & $0.68 \pm 0.20$ & $0.10 \pm 0.02$ & $0.32 \pm 0.03$ & $0.14 \pm 0.02$ & 1.00 \\
\hline $\begin{array}{l}\text { 赤狐 + 黄喉雉敦 Vulpes } \\
\text { vulpes + Tetraophasis szechenyii }\end{array}$ & $0.56 \pm 0.07$ & $0.70 \pm 0.08$ & $0.31 \pm 0.38$ & $0.09 \pm 0.01$ & $0.42 \pm 0.07$ & $0.09 \pm 0.02$ & $1.42 \pm 0.41$ \\
\hline $\begin{array}{l}\text { 赤狐 }+ \text { 血雉 Vulpes vulpes }+ \\
\text { Ithaginis cruentus }\end{array}$ & $0.62 \pm 0.07$ & $0.81 \pm 0.30$ & $0.29 \pm 0.17$ & $0.08 \pm 0.01$ & $0.59 \pm 0.05$ & $0.11 \pm 0.01$ & $1.31 \pm 0.14$ \\
\hline
\end{tabular}

参数含义见表1 The meaning of parameters is shown in Table 1.
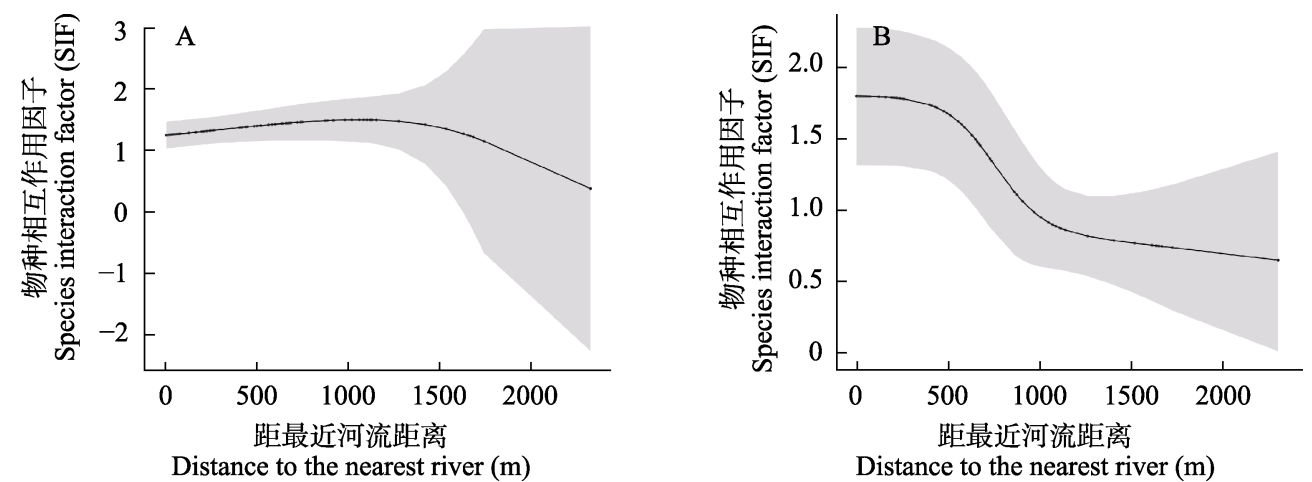

图2 基于最优模型预测的协变量对赤狐与血雉(A)和黄喉雉嚲(B)在位点水平上的物种相互作用因子(SIF)的影响。灰色部分 为每个位点SIF值的 $95 \%$ 置信区间。

Fig. 2 The effect of covariates predicted by the optimal model on the species interaction factor (SIF) of the red fox and blood pheasant (A), red fox and buff-throated partridge (B) at the single site level. Gray area indicates 95\% confidence intervals for SIF value of each site.
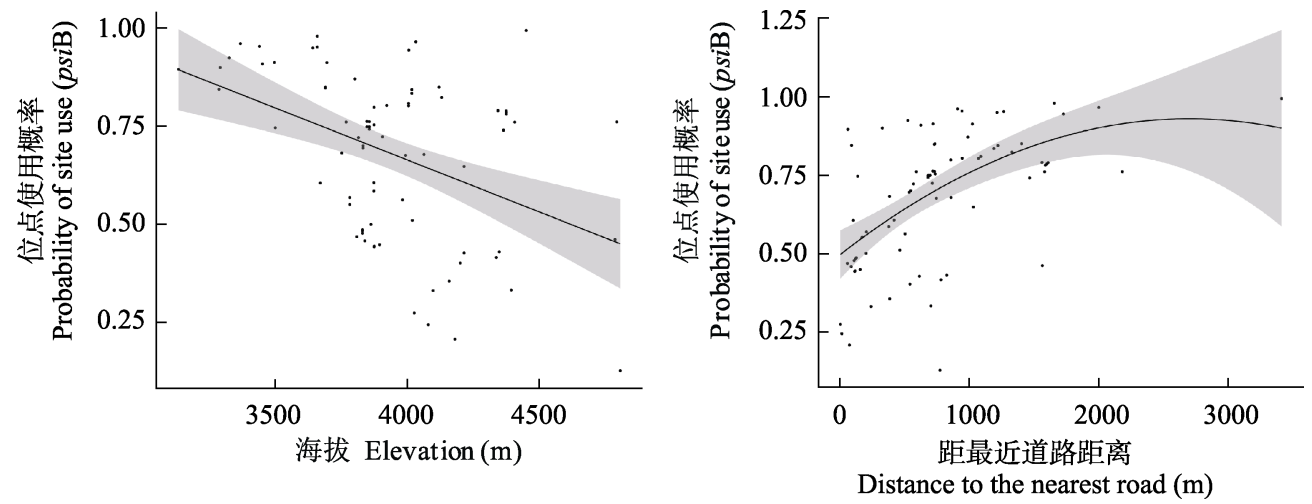

图3 基于最优模型预测的协变量在位点水平上对白马鸡位点使用概率的影响。黑色曲线为多项式回归拟合曲线, 灰色区域 为 $95 \%$ 置信区间。

Fig. 3 The effect of covariates predicted by the optimal model on the white eared-pheasant's site use at the single site level. The black curve fitted with polynomial regression, gray area indicates $95 \%$ confidence intervals.

件型双物种占域模型时不纳入探测协变量。条件型 双物种占域模型结果显示当赤狐存在时 3 种雉类的 探测概率低于赤狐不存在时 $(p \mathrm{~B}>r \mathrm{~B})($ 表3)。既当 赤 狐存在时, 3 种雉类可能会采取更加隐蔽的活动方 式或减少受食强度躲避来自赤狐的捕食风险。

\section{3 讨论}

单物种占域模型结果显示, 距最近河流距离是 影响黄喉雉嚲和血雉对位点使用概率的重要因子。 有研究表明黄喉雉敦和血雉都偏好距水源距离近 的生境(贾陈喜等, 1999; Wang et al, 2021)。已有对白 
马鸡的生境选择研究结果显示, 白马鸡主要偏好于 距离水源近且灌木盖度高的生境(Jia et al, 2005; 贾 非等, 2005), 而本研究结果显示白马鸡偏好于选择 远离道路且海拔相对较低的生境, 对其他因子没有 明显的偏好。差异来源可能是本研究的范围尺度比 已有研究大, 因子更宏观。在这3种雉类中, 白马鸡 的活动海拔相对偏低(Wang et al, 2021), 加之体型 较大, 常集群, 活动范围较大, 对人类活动相对敏 感(格玛嘉措等, 1999), 故其可能要相对远离道路。 同时，由于环境的差异性，同一物种在不同区域本 身就会表现出不同的环境因子偏好(杨春花等, 2006)。本研究结果还显示环境因子不影响赤狐对位 点的使用, 这可能是由于赤狐可栖息的生境类型十 分丰富, 其空间分布主要取决于食物的丰富度(马 勇等, 2014), 受环境因子的影响相对较小。

根据条件型双物种占域模型的结果, 赤狐与 3 种同域分布的雉类物种对的分布关系都受到了环 境变量的影响, 但赤狐只对黄喉雉敦和血雉的位点 使用概率产生影响 $(\mathrm{SIF} \neq 1)$ 。赤狐与黄喉雉鹑和 血雉的空间分布趋于重合(SIF > 1), 且当活动区域 距河流距离越近, 赤狐与黄喉雉敦和血雉的空间分 布重合度越高，也意味着发生捕食行为的概率更 高。有研究表明捕食者偏好在食物丰富的环境中活 动且大多数捕食者具有追踪猎物的能力, 这会导致 捕食者和猎物密度呈正相关关系 (Godin \& Keenleyside, 1984; Luttbeg \& Sih, 2004; Ngoprasert et al, 2012)。黄喉雉敦和血雉都是赤狐捕食的猎物, 且赤狐也偏好距河流较近的生境(Cavallini \& Lovari 1991), 所以其共同出现的概率高。赤狐不影 响白马鸡对位点的使用, 二者的空间分布相互独立 $(\mathrm{SIF}=1)$ 。白马鸡对位点的使用概率随海拔升高而 降低, 随位点距道路距离的升高而升高。有研究表 明在同一区域内, 捕食者与不同猎物的空间分布关 系会受其对猎物偏好的影响，从而与不同猎物表现 出不同的空间分布关系(Steinmetz et al, 2013)。相比 于黄喉雉敦和血雉, 白马鸡体型更大, 可能不是赤 狐最佳的捕食选择, 从而在相机位点表现出与黄喉 雉㕫和血雉有更高的概率共同出现，与白马鸡在空 间分布上相互独立。

在占域模型中, 一个物种可能改变另一物种的 探测概率，进而影响它们在整个景观中的占域概率
(Bailey et al, 2009)。捕食者会在一定程度上影响猎 物的探测概率(Wang et al, 2015; Farris et al, 2016; Murphy et al, 2019)。本研究结果也显示当赤狐分别 与 3 种雉类使用同一位点时, 3种雉类的探测概率因 赤狐的存在而下降, 且3个物种对的探测概率仅受 物种作用的影响, 不受环境因子影响。猎物可能会 避开捕食者最可能出现的地点而选择更加隐蔽复 杂的栖息地或是减少自身活动时间来减少与捕食 者相遇的几率(Werner, 1992; Creel \& Winnie, 2005)。 尽管赤狐与黄喉雉敦和血雉在空间分布上趋于重 合, 与白马鸡在空间分布上相互独立, 但 3 种雉类 都可能会在赤狐存在的区域中避开赤狐活动最活 跃的地点或是调整自身活动时间和微生境来避开 赤狐的捕食, 从而降低了 3 种雉类的探测率。

本研究结果显示捕食作用与环境因子促使赤 狐与黄喉雉敦和血雉在相机位点有更高的概率共 同出现, 而赤狐与白马鸡的空间关系只受环境因子 影响。同时捕食作用也降低了 3 种雉类的探测概率。

\section{参考文献}

Bailey LL, Reid JA, Forsman ED, Nichols JD (2009) Modeling co-occurrence of northern spotted and barred owls: Accounting for detection probability differences. Biological Conservation, 142, 2983-2989.

Burton AC, Neilson E, Moreira D, Ladle A, Steenweg R, Fisher JT, Bayne E, Boutin S (2015) Wildlife camera trapping: A review and recommendations for linking surveys to ecological processes. Journal of Applied Ecology, 52, 675-685.

Case TJ, Bolger DT (1991) The role of introduced species in shaping the distribution and abundance of island reptiles. Evolutionary Ecology, 5, 272-290.

Cavallini P, Lovari S (1991) Environmental-factors influencing the use of habitat in the red fox, Vulpes vulpes. Journal of Zoology, 223, 323-339.

Chen LJ, Shu ZF, Yao WT, Ma Y, Xiao WH, Huang XQ (2019) Combined effects of habitat and interspecific interaction define co-occurrence patterns of sympatric Galliformes. Avian Research, 10, 344-356.

Chen YH, Luiselli L (2009) Species richness and co-occurrence patterns of Galliformes in China at three large spatial scales: Does scale size matter? Revue D Ecologie-la Terre Et La Vie, 64, 251-260.

Connell JH (1983) On the prevalence and relative importance of interspecific competition: Evidence from field experiments. The American Naturalist, 122, 661-696.

Creel S, Winnie JA Jr (2005) Responses of elk herd size to fine-scale spatial and temporal variation in the risk of 
predation by wolves. Animal Behaviour, 69, 1181-1189.

D’Amen M, Mod HK, Gotelli NJ, Guisan A (2018) Disentangling biotic interactions, environmental filters, and dispersal limitation as drivers of species co-occurrence. Ecography, 41, 1233-1244.

Deng L, Yang HZ (2010) Estimation of natural ecological value for the plateau pasturing area-A case study in Xinlong County of Sichuan Province. Sichuan Environment, 29(2), 89-94. (in Chinese with English abstract) [邓林, 杨 海真 (2010) 高原牧区自然生态价值的测算——四川 省甘孜藏族自治州新龙县为例. 四川环境, 29(2), 89-94.]

Farris ZJ, Kelly MJ, Karpanty S, Ratelolahy F (2016) Patterns of spatial co-occurrence among native and exotic carnivores in north-eastern Madagascar. Animal Conservation, 19, 189-198.

Ge MJC, Dong DF, Long WX (1999) A preliminary observation on the ecological habits of white eared-pheasant. Chinese Journal of Zoology, 34(1), 26-28. (in Chinese) [格玛嘉措, 董德福, 龙文祥 (1999) 白马鸡 生态习性的初步观察. 动物学杂志, 34(1), 26-28.]

Godin JGJ, Keenleyside MHA (1984) Foraging on patchily distributed prey by a cichlid fish (Teleostei, Cichlidae): A test of the ideal free distribution theory. Animal Behaviour, 32, 120-131.

Hines JE (2006) PRESENCE-Software to estimate patch occupancy and related parameters. USGS-PWRC. http://www.mbr-pwrc.usgs.gov/software. (accessed on 202011-25)

Jia CX, Zheng GM, Zhou XP, Zhang HM (1999) Social organization of blood pheasant (Ithaginis cruentus) in Wolong Nature Reserve. Acta Zoologica Sinica, 45, 135-142. (in Chinese with English abstract) [贾陈喜, 郑光 美, 周小平, 张和民 (1999) 卧龙自然保护区血雉的社群 组织. 动物学报, 45, 135-142.]

Jia F, Wang N, Zheng GM (2005) Winter habitat requirements of white eared-pheasant Crossoptilon crossoptilon and blood pheasant Ithaginis cruentus in south-west China. Bird Conservation International, 15, 303-312.

Jia F, Wang N, Zheng GM (2005) Habitat selection and spatial distribution of white eared-pheasant Crossoptilon crossoptilon during early breeding period. Acta Zoologica Sinica, 51, 383-392. (in Chinese with English abstract) [贾 非, 王楠, 郑光美 (2005) 白马鸡繁殖早期栖息地选择和 空间分布. 动物学报, 51, 383-392.]

Lesmeister DB, Nielsen CK, Schauber EM, Hellgren EC (2015) Spatial and temporal structure of a mesocarnivore guild in midwestern North America. Wildlife Monographs, 191, 1-61.

Li LY, Wang HD, Zhang H, Teng LW, Liu ZS (2014) Research progress of habitat selection by red fox (Vulpes vulpes). Journal of Anhui Agricultural Sciences, 42, 3289-3292, 3295. (in Chinese with English abstract) [李路云, 王海东, 张海, 滕丽微, 刘振生 (2014) 赤狐生境选择研究进展.
安徽农业科学, 42, 3289-3292, 3295.]

Luttbeg B, Sih A (2004) Predator and prey habitat selection games: The effects of how prey balance foraging and predation risk. Israel Journal of Zoology, 50, 233-254.

Ma Y, Sun ZH, Liu ZS, Teng LW (2014) Food habits by red fox (Vulpes vulpes), a review. Journal of Economic Animal, 18, 53-58. (in Chinese with English abstract) [马勇, 孙兆 惠, 刘振生, 滕丽微 (2014) 赤狐食性的研究进展. 经济 动物学报, 18, 53-58.]

MacKenzie DI, Bailey LL, Nichols JD (2004) Investigating species co-occurrence patterns when species are detected imperfectly. Journal of Animal Ecology, 73, 546-555.

Magle SB, Simoni LS, Lehrer EW, Brown JS (2014) Urban predator-prey association: Coyote and deer distributions in the Chicago metropolitan area. Urban Ecosystems, 17, 875-891.

Murphy A, Kelly MJ, Karpanty SM, Andrianjakarivelo V, Farris ZJ (2019) Using camera traps to investigate spatial co-occurrence between exotic predators and native prey species: A case study from northeastern Madagascar. Journal of Zoology, 307, 264-273.

Ngoprasert D, Lynam AJ, Sukmasuang R, Tantipisanuh N, Chutipong W, Steinmetz R, Jenks KE, Gale GA, Grassman LI Jr, Kitamura S, Howard J, Cutter P, Cutter P, Leimgruber P, Songsasen N, Reed DH (2012) Occurrence of three felids across a network of protected areas in Thailand: Prey, intraguild, and habitat associations. Biotropica, 44, 810-817.

Rabelo RM, Aragón S, Bicca-Marques JC (2019) Prey abundance drives habitat occupancy by jaguars in Amazonian floodplain river islands. Acta Oecologica, 97, 28-33.

Richmond OMW, Hines JE, Beissinger SR (2010) Two-species occupancy models: A new parameterization applied to co-occurrence of secretive rails. Ecological Applications, 20, 2036-2046.

Ritchie EG, Martin JK, Johnson CN, Fox BJ (2009) Separating the influences of environment and species interactions on patterns of distribution and abundance: Competition between large herbivores. Journal of Animal Ecology, 78, 724-731.

Steinmetz R, Seuaturien N, Chutipong W (2013) Tigers, leopards, and dholes in a half-empty forest: Assessing species interactions in a guild of threatened carnivores. Biological Conservation, 163, 68-78.

Wang B, Xu Y, Price M, Yang N, Liu W, Zhu BW, Zhong X, Ran JH (2021) Niche partitioning among three montane ground-dwelling pheasant species along multiple ecological dimensions. Ibis, 163, 171-182.

Wang YW, Allen ML, Wilmers CC (2015) Mesopredator spatial and temporal responses to large predators and human development in the Santa Cruz Mountains of California. Biological Conservation, 190, 23-33.

Werner EE (1992) Individual behavior and higher-order species 
interactions. The American Naturalist, 140, s5-s32.

Yang CH, Zhang HM, Zhou XP, Wang PY, Wang XM (2006) Review of habitat selection in the giant panda (Ailuropoda melanoleuca). Acta Ecologica Sinica, 26, 3442-3453. (in Chinese with English abstract) [杨春花, 张和民, 周小平, 王鹏彦, 王小明 (2006) 大熊猫(Ailuropoda melanoleuca) 生境选择研究进展. 生态学报, 26, 3442-3453.]

You ZQ, Tang ZH, Yang YB, Yang LH, Shi HY, Liu H, Gan $X$, Zheng TC, Jiang ZG (2014) Summer habitat selection by white-lipped deer (Cervus albirostris) in Chaqingsongduo White-lipped Deer National Nature Reserve. Acta Theriologica Sinica, 34, 46-53. (in Chinese with English abstract) [游章强, 唐中海, 杨远斌, 杨丽红, 石红艳, 刘 吴, 甘波, 郑天才, 蒋志刚 (2014) 察青松多白唇鹿国家 级自然保护区白唇鹿对夏季生境的选择. 兽类学报, 34, 46-53.]

Zhao GJ, Gong YN, Yang HT, Xie B, Wang TM, Ge JP, Feng
LM (2019) Study on habitat use and activity rhythms of wild boar in eastern region of Northeast Tiger and Leopard National Park. Acta Theriologica Sinica, 39, 431-441. (in Chinese with English abstract) [赵国静, 宫一男, 杨海涛, 谢冰，王天明，葛剑平，冯利民 (2019) 东北虎豹国家公 园东部的野猪生境利用和活动节律初步研究. 兽类学报, 39, 431-441.]

Zhu BW, Wang B, Ran JH, Li B, Huang F, Li XQ, Gu XD (2019) Seasonal variation of daily activity patterns and diet of yellow-throated marten (Martes flavigula). Acta Theriologica Sinica, 39, 52-61. (in Chinese with English abstract) [朱博伟, 王彬, 再江洪, 李波, 黄蜂, 李晓清, 古晓东 (2019) 黄喉貂日活动节律及食性的季节变化. 兽类学报, 39, 52-61.]

(责任编委: 李晟 责任编辑: 问文杰)

\section{附录 Supplementary Material}

\section{附录 1 赤狐与 3 种雉类的 8 种条件型双物种占域模型的候选模型}

Appendix 1 Eight candidate conditional two-species occupancy model for red fox and three pheasant species https://www.biodiversity-science.net/fileup/PDF/2020438-1.pdf

附录2 4个物种单物种占域模型选择结果

Appendix 2 Selection results of single species occupancy model for four target species https://www.biodiversity-science.net/fileup/PDF/2020438-2.pdf 
邹博研, 罗概, 朱博伟, 苒江洪, 房超 (2021) 川西高原三种雉类与其捕食者赤狐的空间关系. 生物多样性, 29, 918-926. https://www.biodiversity-science.net/CN/10.17520/biods.2020438

附录 1 赤狐与 3 种雉类的 8 种条件型双物种占域模型的候选模型

Appendix 1 Eight candidate conditional two-species occupancy model for red fox and three pheasant species

\begin{tabular}{|c|c|}
\hline 模型类型 Types of models & 假设 Hypothesis \\
\hline$p s i \mathrm{~A}, p s i \mathrm{~B} ; p \mathrm{~A}, p \mathrm{~B}$ & $\begin{array}{l}\text { 环境变量和捕食者存在对于雉类的位点使用和探测都无影响 } \\
\text { Neither habitat nor predator presence influences pheasants' site } \\
\text { use/detection }\end{array}$ \\
\hline$p s i \mathrm{~A}, p s i \mathrm{~B} ; p \mathrm{~A}, p \mathrm{~B}, r \mathrm{~B}$ & $\begin{array}{l}\text { 仅捕食者的存在对雉类的探测有影响 Only predator presence } \\
\text { influences pheasants' detection }\end{array}$ \\
\hline$p s i \mathrm{~A}, p s i \mathrm{BA}, p s i \mathrm{Ba} ; p \mathrm{~A}, p \mathrm{~B}$ & $\begin{array}{l}\text { 仅捕食者的存在对雉类的位点使用有影响 Only predator presence } \\
\text { influences pheasants' site use }\end{array}$ \\
\hline$p s i \mathrm{~A}, p s i \mathrm{BA}, p s i \mathrm{Ba} ; p \mathrm{~A}, p \mathrm{~B}, r \mathrm{~B}$ & $\begin{array}{l}\text { 捕食者的存在对雉类的位点使用和探测都有影响 Only predator } \\
\text { presence influences pheasants' site use/detection }\end{array}$ \\
\hline$p s i \mathrm{~A}(\mathrm{H}), p s i \mathrm{~B}(\mathrm{H}) ; p \mathrm{~A}(\mathrm{H}), p \mathrm{~B}(\mathrm{H})$ & $\begin{array}{l}\text { 只有环境变量影响雉类的位点使用和探测 Only habitat influences } \\
\text { pheasants' site use/detection }\end{array}$ \\
\hline$p s i \mathrm{~A}(\mathrm{H}), p s i \mathrm{~B}(\mathrm{H}) ; p \mathrm{~A}(\mathrm{H}), p \mathrm{~B}(\mathrm{H}), r \mathrm{~B}(\mathrm{H})$ & $\begin{array}{l}\text { 环境变量介导的捕食者的存在对雉类的探测有影响 Habitat- } \\
\text { mediated influence of predator presence on pheasants' detection }\end{array}$ \\
\hline$p s i \mathrm{~A}(\mathrm{H}), p s i \mathrm{BA}(\mathrm{H}), p s i \mathrm{Ba}(\mathrm{H}) ; p \mathrm{~A}(\mathrm{H}), p \mathrm{~B}(\mathrm{H})$ & $\begin{array}{l}\text { 环境变量介导的捕食者的存在对雉类的位点使用有影响 Habitat- } \\
\text { mediated influence of predator presence on pheasants' site use }\end{array}$ \\
\hline $\begin{array}{l}p s i \mathrm{~A}(\mathrm{H}), p s i \mathrm{BA}(\mathrm{H}), p s i \mathrm{Ba}(\mathrm{H}) ; p \mathrm{~A}(\mathrm{H}), p \mathrm{~B}(\mathrm{H}), \\
r \mathrm{~B}(\mathrm{H})\end{array}$ & $\begin{array}{l}\text { 环境变量介导的捕食者的存在对雉类的位点使用和探测都有影响 } \\
\text { Habitat-mediated influence of predator presence on pheasants'site } \\
\text { use/detection }\end{array}$ \\
\hline
\end{tabular}


邹博研，罗概，朱博伟，苒江洪，房超 (2021) 川西高原三种雉类与其捕食者赤狐的空间关系. 生物多样性, 29, 918-926. https://www.biodiversity-science.net/CN/10.17520/biods.2020438

附录2 4个物种单物种占域模型选择结果

Appendix 2 Selection results of single species occupancy model for four target species

\begin{tabular}{|c|c|c|c|c|c|}
\hline 物种 Species & 模型 Models & $\begin{array}{l}\text { 参数数量 } \\
\text { Number of } \\
\text { parameters }\end{array}$ & AIC & $\Delta \mathrm{AIC}$ & AIC Wt \\
\hline \multicolumn{6}{|l|}{$\begin{array}{l}\text { 赤狐 Vulpes } \\
\text { vulpes }\end{array}$} \\
\hline & psi (.);p (.) & 2 & 362.02 & 0.00 & 0.1381 \\
\hline & $p s i(\mathrm{DTR}) ; P()$. & 3 & 363.51 & 1.49 & 0.0656 \\
\hline & $p s i(\mathrm{SLO}) ; p()$. & 3 & 363.67 & 1.65 & 0.0604 \\
\hline & psi (EVI); $p()$. & 3 & 363.91 & 1.90 & 0.0535 \\
\hline \multicolumn{6}{|l|}{$\begin{array}{l}\text { 黄喉雉敦 } \\
\text { Tetaophasis } \\
\text { szechenyii }\end{array}$} \\
\hline & $p s i(\mathrm{DTW}+\mathrm{EVI}) ; p()$. & 4 & 459.97 & 0.00 & 0.0533 \\
\hline & psi (DTW); $p()$. & 3 & 460.66 & 0.69 & 0.0377 \\
\hline & $p s i(\mathrm{ASP}+\mathrm{DTW}+\mathrm{EVI}) ; p()$. & 5 & 461.20 & 1.23 & 0.0289 \\
\hline & $p s i(\mathrm{ASP}+\mathrm{DTW}) ; p()$. & 4 & 461.35 & 1.37 & 0.0268 \\
\hline & $p s i(\mathrm{DTW}+\mathrm{ELE}+\mathrm{EVI}) ; p()$. & 5 & 461.59 & 1.62 & 0.0237 \\
\hline & $p s i(\mathrm{ELE}+\mathrm{EVI}) ; p()$. & 4 & 461.81 & 1.84 & 0.0213 \\
\hline \multicolumn{6}{|l|}{$\begin{array}{l}\text { 血雉 Ithaginis } \\
\text { cruentus }\end{array}$} \\
\hline & $p s i(\mathrm{DTW}) ; p()$. & 3 & 607.38 & 0.00 & 0.0918 \\
\hline & $p s i(\mathrm{DTR}+\mathrm{DTW}) ; p()$. & 4 & 608.12 & 0.74 & 0.0633 \\
\hline & $p s i(\mathrm{DTW}+\mathrm{EVI}) ; p()$. & 4 & 608.33 & 0.95 & 0.0572 \\
\hline & $p s i(\mathrm{ASP}+\mathrm{DTW}) ; p()$. & 4 & 608.95 & 1.56 & 0.0420 \\
\hline & $p s i(\mathrm{DTW}+\mathrm{PRE}) ; p()$. & 4 & 609.38 & 1.99 & 0.0338 \\
\hline \multicolumn{6}{|l|}{$\begin{array}{l}\text { 白马鸡 } \\
\text { Crossoptilon } \\
\text { crossoptilon }\end{array}$} \\
\hline & $p s i(\mathrm{DTR}+\mathrm{ELE}) ; p()$. & 4 & 770.85 & 0.00 & 0.0736 \\
\hline & $p s i(\mathrm{DTR}+\mathrm{ELE}+\mathrm{PRE}) ; p()$. & 5 & 771.43 & 0.57 & 0.0553 \\
\hline & $p s i(\mathrm{DTR}+\mathrm{ELE}+\mathrm{EVI}+\mathrm{PRE}) ; p()$. & 6 & 771.48 & 0.63 & 0.0538 \\
\hline & $p s i(\mathrm{DTR}+\mathrm{ELE}+\mathrm{EVI}) ; p()$. & 5 & 771.74 & 0.89 & 0.0472 \\
\hline & $p s i(\mathrm{EVI}+\mathrm{PRE}) ; p()$. & 4 & 772.25 & 1.40 & 0.0366 \\
\hline & $p s i(\mathrm{DTR}+\mathrm{EVI}+\mathrm{PRE}) ; p()$. & 5 & 772.79 & 1.94 & 0.0279 \\
\hline
\end{tabular}

DTR: 距最近公路距离; SLO: 坡度; EVI: 增强型植被指数; DTW：距最近河流距离; ASP: 坡向; ELE: 海拔; PRE: 其他捕 食者RAI。

DTR, Distance to the nearest road; SLO, Slope; EVI, Enhanced vegetation index; DTW, Distance to the nearest river; ASP,

Aspect; ELE, Elevation; PRE, The other predators' RAI. 